\title{
A population-based recurrence risk management study of patients with pT1 node-negative HER2+ breast cancer: a National Clinical Database study
}

\author{
Makoto Kubo $^{1}$ (1) $\cdot$ Masaaki Kawai $^{2} \cdot$ Hiraku Kumamaru $^{3} \cdot$ Hiroaki Miyata $^{3} \cdot$ Kenji Tamura $^{4} \cdot$ Masayuki Yoshida $^{5}$. \\ Etsuyo Ogo ${ }^{6} \cdot$ Masayuki Nagahashi $^{7}$. Sota Asaga ${ }^{8}$. Yasuyuki Kojima ${ }^{9} \cdot$ Takayuki Kadoya $^{10} \cdot$ Kenjiro Aogi $^{11}$. \\ Naoki Niikura ${ }^{12} \cdot$ Minoru Miyashita $^{13} \cdot$ Kotaro lijima $^{14} \cdot$ Naoki Hayashi $^{15} \cdot$ Yutaka Yamamoto $^{16}$. Shigeru Imoto ${ }^{17}$. \\ Hiromitsu Jinno ${ }^{18}$
}

Received: 12 August 2019 / Accepted: 19 August 2019 / Published online: 26 August 2019

(c) The Author(s) 2019

\begin{abstract}
Purpose Recurrence risk management of patients with small $(\leq 2 \mathrm{~cm})$, node-negative, human epidermal growth factor receptor 2 (HER2)-positive breast cancer remains challenging. We studied the effects of adjuvant chemotherapy and/or trastuzumab and survival outcomes among these patients, using data from the population-based Japanese National Clinical Database (NCD).

Methods We identified a cohort of 2736 breast cancer patients with HER2+pT1N0 disease: 489 pT1a, 642 pT1b, and 1623 pT1c. The median observation period was 76 months, and the 5-year follow-up rate was $48.2 \%$. The number of events was 212 for disease-free survival (DFS), 40 for breast cancer-specific survival, and 84 for overall survival (OS).

Results There were $24.5 \%$ of pT1a, $51.9 \%$ of pT1b, and $63.3 \%$ of pT1c patients who were treated systemically after surgery. OS in pT1b (logrank test; $p=0.03$ ) and DFS in pT1c (logrank test; $p<0.001$ ) were significantly improved in treated compared with untreated patients. In the Cox proportional hazards model, treated patients had significantly longer OS than untreated patients in pT1b (hazard ratio (HR) 0.20) and pT1c (HR 0.54) groups. Estrogen receptor-negative tumors was also a significant predictor of survival in pT1c (HR 2.01) but not pT1ab patients. Furthermore, HR was greater in patients aged $\leq 35$ years (3.18) compared to that in patients aged 50-69 years in the pT1b group.

Conclusions NCD data revealed that systemic treatment improved OS in pT1bc but not in pT1a node-negative HER2+ breast cancer patients. Future observational research using big-sized data is expected to play an important role in optimizing treatment for patients with early-stage breast cancer.
\end{abstract}

Keywords Breast cancer $\cdot$ Stage I $\cdot$ Human epidermal growth factor receptor $2 \cdot$ Chemotherapy $\cdot$ Trastuzumab

$\begin{array}{ll}\text { Abbreviations } \\ \text { HER2 } & \text { Human epidermal growth factor receptor } 2 \\ \text { BCR } & \text { Breast Cancer Registry } \\ \text { NCD } & \text { National Clinical Database } \\ \text { JBCS } & \text { Japanese Breast Cancer Society } \\ \text { IHC } & \text { Immunohistochemistry } \\ \text { ER } & \text { Estrogen receptor }\end{array}$

Electronic supplementary material The online version of this article (https://doi.org/10.1007/s10549-019-05413-7) contains supplementary material, which is available to authorized users.

Makoto Kubo

mkubo@tumor.med.kyushu-u.ac.jp

Extended author information available on the last page of the article

$\begin{array}{ll}\text { PR } & \text { Progesterone receptor } \\ \text { DFS } & \text { Disease-free survival } \\ \text { BCSS } & \text { Breast cancer-specific survival } \\ \text { OS } & \text { Overall survival } \\ \text { HR } & \text { Hazard ratio } \\ \text { CI } & \text { Confidence interval } \\ \text { NCCN } & \text { National comprehensive cancer network } \\ \text { vs. } & \text { Versus } \\ \text { RFS } & \text { Recurrence-free survival } \\ \text { AIs } & \text { Aromatase inhibitors } \\ \text { T-DM1 } & \text { Trastuzumab-emtansine }\end{array}$

PR Progesterone receptor

BCSS Breast cancer-specific survival

OS Overall survival

HR Hazard ratio

CI Confidence interval

NCCN National comprehensive cancer network

vs. Versus

$\begin{array}{ll}\text { AIs } & \text { Aromatase inhibitors } \\ \text { T-DM1 } & \text { Trastuzumab-emtansine }\end{array}$ 


\section{Introduction}

The incidence of stage I breast cancer has increased gradually to nearly half of all primary breast cancer cases because of detection of nonpalpable breast cancer using screening mammography in Japan as well as in Europe and the United States [1-5]. According to breast cancer registry, there was a marked increase from 37.3 to $51.6 \%$ in the proportion of stage I breast cancer patients during the year 2004 to 2014 [1]. Although it is well known that patients with T1ab nodenegative tumors ( $1 \mathrm{~cm}$ or less) generally have a favorable prognosis, outcomes for those patients may depend largely on tumor subtypes [6,7].

Human epidermal growth factor receptor 2 (HER2) overexpression is an independent, poor prognostic factor, and a positive predictive biomarker in response to HER2-targeted therapy [8]. HER2-overexpressing or HER2-amplified breast cancer accounts for $20-30 \%$ of invasive breast carcinomas [8], and without treatment, has the worst prognosis among subtypes [7]. Various guidelines support the use of trastuzumab-based chemotherapy as a standard option in tumors larger than $1 \mathrm{~cm}$, and suggest its administration in T1b tumors because some studies have reported that HER2 overexpression was an independent, poor prognostic factor even for patients with pT1ab node-negative HER2+ breast cancer $[6,9]$.

Trastuzumab (Herceptin ${ }^{\circledR}$, Roche) has been available as adjuvant systemic therapy in Japan since February 2008. Several randomized clinical trials [10-16] have shown an improved outcome among Trastuzumab users in pStage II-III disease. However, since patients with pStage I disease were mostly excluded from these trials, the evidence for benefit of chemotherapy and/or trastuzumab in those patients is limited. The aim of our study was to assess the recurrence and survival of pT1abN0 HER2+ breast cancer patients across different types of treatments and hormone receptor types, using data from the nationwide breast cancer registry, to provide information on possible treatment options to the healthcare providers.

\section{Patients and methods}

\section{Data collection}

The Breast Cancer Registry (BCR) run on the National Clinical Database (NCD) contains records of more than 560,000 patients with breast cancer from more than 1400 hospitals, as of 2015. Affiliated institutions provide data on newly diagnosed primary breast cancer patients through a web-based system to the BCR-NCD, covering more than 50 demographic and clinicopathologic characteristics. The initial follow-up is requested for 5-year prognosis since first treatment (preoperative therapy or surgery). The BCR-NCD was originally maintained by the Registration Committee of the Japanese Breast Cancer Society (JBCS) and was supported by the Public Health Research Foundation (Tokyo, Japan). It is currently managed by NCD, which is a platform for various nationwide registries in Japan. TNM classification is now registered according to the 7th edition of the Unio Internationalis Contra Cancrum staging system [17], and histological classification was registered according to the General Rules for Clinical and Pathological Recording of Breast Cancer [18], which was further transferred to the Classification of Tumors of the Breast and Female Genital Organs [19].

\section{Study patients}

For our study, among 238,711 breast cancer patients registered between 2004 and 2011, we selected 186,059 female patients who underwent surgery (Fig. 1). Patients with bilateral tumors, those who received preoperative systemic therapy, and those with distant metastases were excluded, resulting in 55,917 patients. HER2 overexpression was defined as immunohistochemistry (IHC) $3+$ and/or a positive fluorescent in situ hybridization test according to the manufacturer's criteria [20]. Hormone receptor (estrogen receptor (ER) or progesterone receptor (PR)) expression was considered positive if at least $1 \%$ of nuclei in tumor cells were stained using IHC for ER or PR. Tumor subtypes were categorized on the basis of IHC as follows: luminal A (ER+, PR $\geq 20 \%$, and HER2-); luminal B (ER+, PR $<20 \%$, and HER2-) [21-23]; luminal-HER2 (ER+and/or PR+/ HER2+); HER2 (ER - and PR-/HER2+); and triple negative (ER-, PR-, and HER2-). Of the 49,458 patients with hormone receptor and HER2 expression-known pT1 tumors registered in the NCD, 21,603 patients (46.3\%) with 5-year follow-up data were taken up for the study and analyzed for survivals based on different subtypes (Cohort 1).

\section{Exposure and patient cohort}

Of the 6339 patients with pT1 HER2+tumors, 623 tumors were removed because of receiving preoperative treatment, and an additional 45 cases were removed because of a lack of treatment information, leaving 2736 patients (48.2\%) with 5-year follow-up data, who were analyzed in our study (Cohort 2). Of the patients, we compared 1472 treated patients by systemic therapies (chemotherapy with or without trastuzumab) to 1265 untreated patients. 
Fig. 1 Study flow chart

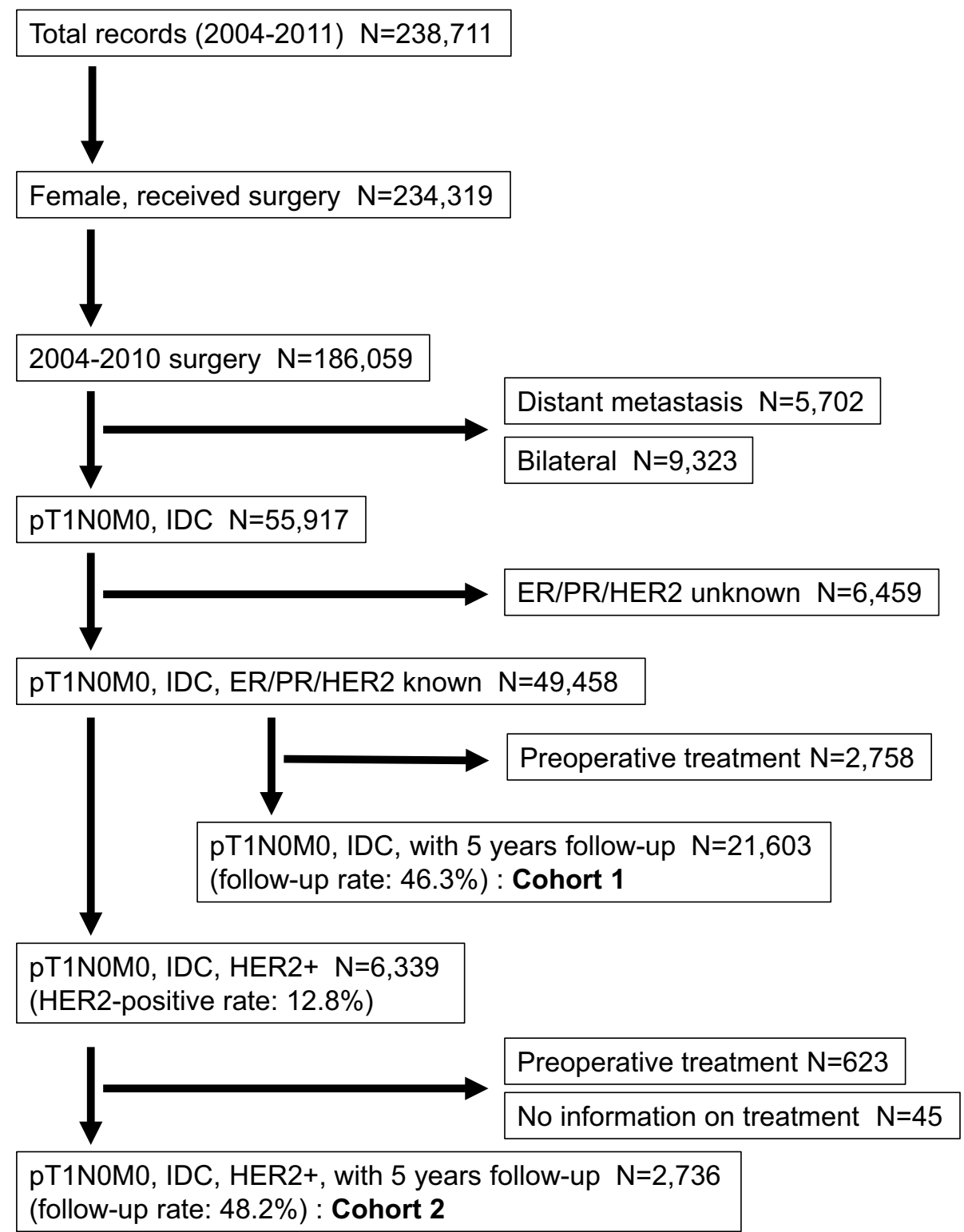

\section{Statistical analysis}

Pearson's Chi squared test was used to determine differences among the three patient groups, all with T1/HER2+ status. Wilcoxon's test were used for intergroup comparisons of continuous variables.

Survival curves were constructed using the Kaplan-Meier method with and without stratification on known prognostic factors and were compared using logrank test. Multivariable survival analyses for disease-free survival (DFS), breast cancer-specific survival (BCSS), and overall survival (OS) were performed using Cox proportional hazards modeling, to estimate the hazard of systemically treated patients relative to untreated patients by pT1a-c group. We considered the following variables as potential confounders in the Cox model: age, surgery, ER and PR statuses, postoperative systemic therapy, and pathological type. Patients with any missing or unknown data were excluded from these analyses. DFS was defined as the time interval between the date of surgery and the time of local or distant recurrence. BCSS and OS were defined as the time interval between the date of surgery and the date of breast cancer-related death or death from any cause, respectively. All tests were two-sided and $a p$ value $<0.05$ was considered statistically significant. All analyses were carried out using SAS version 9.4 (SAS Institute, Cary, NC). 


\section{Results}

We identified a cohort of 2736 patients with HER2+ pT1N0M0 disease (Fig. 1). Patient characteristics are shown in Table 1. The median follow-up period was 76 months, and the 5 -year follow-up rate was $48.2 \%$. The number of events was 212 for DFS, 40 for BCSS, and 84 for OS.

In general, patients with T1c tumors had a poorer OS compared to those with T1a or T1b tumors. Among patients with HER2+ tumors, those with T1b or T1c tumors had a poorer OS compared to those with T1a tumors (Supplementary Fig. 1). Moreover, patients with luminal A and luminalHER2 T1c tumors did not have poorer OS, whereas patients with luminal B and triple negative T1c tumors had significantly poorer OS compared to those with $\mathrm{T} 1 \mathrm{a}$ or $\mathrm{T} 1 \mathrm{~b}$ tumors
(Supplementary Fig. 2). Patients with HER2-enriched T1c and also T1b tumors had a poorer OS compared to those with T1a tumors $(p=0.04)$.

Prognostic information was available for $489 \mathrm{~T} 1 \mathrm{a}$ (17.9\%), $624 \mathrm{~T} 1 \mathrm{~b}(22.8 \%)$, and $1623 \mathrm{~T} 1 \mathrm{c}$ patients (59.3\%) (Table 1). There were $24.5 \%$ of $\mathrm{T} 1 \mathrm{a}, 51.9 \%$ of $\mathrm{T} 1 \mathrm{~b}$, and $63.3 \%$ of T1c patients who received treatment, chemotherapy, and/or trastuzumab. HER $2+$ patients aged $<50$, those who were premenopausal, those who received breastconserving surgery, and those who were ER-/PR- had a high probability of receiving systemic treatment, while patients with $\mathrm{ER}+\mathrm{T} 1 \mathrm{~b}$ tumors were less likely to be treated. Those with ER- compared with ER+tumors tended to be treated with chemotherapy and/or trastuzumab to a greater extent (Supplementary Fig. 3A). The treatment group in the late phase (2008-2010) compared with the early phase (2004-2007) decreased by $6.8 \%$, resulting in $23.2 \%$ for T1a;

Table 1 Clinicopathologic characteristics of pT1N0 HER2-positive breast cancer (cohort 2)

\begin{tabular}{|c|c|c|c|c|c|c|}
\hline & \multicolumn{2}{|l|}{ pT1a } & \multicolumn{2}{|l|}{ pT1b } & \multicolumn{2}{|l|}{ pT1c } \\
\hline & No treatment & Treatment & No treatment & Treatment & No treatment & Treatment \\
\hline$N$ & 369 & 120 & 300 & 324 & 595 & 1028 \\
\hline \multicolumn{7}{|l|}{ Age at diagnosis } \\
\hline$<35$ & $10(2.7 \%)$ & $7(5.8 \%)$ & $9(3.0 \%)$ & $14(4.3 \%)$ & $15(2.5 \%)$ & $39(3.8 \%)$ \\
\hline $35-49$ & $79(21.4 \%)$ & $44(36.7 \%)$ & $69(23.0 \%)$ & $89(27.5 \%)$ & $124(20.8 \%)$ & $256(24.9 \%)$ \\
\hline $50-69$ & $240(65.0 \%)$ & $68(56.7 \%)$ & $172(57.3 \%)$ & $198(61.1 \%)$ & $296(49.8 \%)$ & $646(62.8 \%)$ \\
\hline$\geq 70$ & $40(10.8 \%)$ & $1(0.8 \%)$ & $50(16.7 \%)$ & $23(7.1 \%)$ & $160(26.9 \%)$ & $87(8.5 \%)$ \\
\hline \multicolumn{7}{|l|}{ Operation } \\
\hline BCS & $165(44.7 \%)$ & $45(37.5 \%)$ & $204(68.0 \%)$ & $185(57.1 \%)$ & $395(66.4 \%)$ & $669(65.1 \%)$ \\
\hline Mastectomy & $204(55.3 \%)$ & $74(61.7 \%)$ & $94(31.3 \%)$ & $137(42.3 \%)$ & $197(33.1 \%)$ & $357(34.7 \%)$ \\
\hline Other & 0 & $1(0.8 \%)$ & $2(0.7 \%)$ & $2(0.6 \%)$ & $3(0.5 \%)$ & $2(0.2 \%)$ \\
\hline \multicolumn{7}{|l|}{ ER } \\
\hline Positive & $149(40.4 \%)$ & $29(24.2 \%)$ & $212(70.7 \%)$ & $141(43.5 \%)$ & $447(75.1 \%)$ & $516(50.2 \%)$ \\
\hline Negative & $220(59.6 \%)$ & $91(75.8 \%)$ & $88(29.3 \%)$ & $183(56.5 \%)$ & $148(24.9 \%)$ & $512(49.8 \%)$ \\
\hline \multicolumn{7}{|l|}{ PR } \\
\hline Positive & $87(23.6 \%)$ & $17(14.2 \%)$ & $143(47.7 \%)$ & $89(27.5 \%)$ & $324(54.5 \%)$ & $339(33.0 \%)$ \\
\hline Negative & $282(76.4 \%)$ & $103(85.8 \%)$ & $157(52.3 \%)$ & $235(72.5 \%)$ & $271(45.6 \%)$ & $689(67.0 \%)$ \\
\hline \multicolumn{7}{|l|}{ Treatment } \\
\hline Chemotherapy alone & & $58(11.9 \%)$ & & $136(21.8 \%)$ & & $384(23.7 \%)$ \\
\hline Trastuzumab alone & & $23(4.7 \%)$ & & $45(7.2 \%)$ & & $83(5.1 \%)$ \\
\hline Both* & & $39(8.0 \%)$ & & $143(22.9 \%)$ & & $561(34.6 \%)$ \\
\hline No treatment & $369(75.5 \%)$ & & $300(48.1 \%)$ & & $595(36.7 \%)$ & \\
\hline \multicolumn{7}{|l|}{ Year of operation } \\
\hline 2004-2005 & $56(15.2 \%)$ & $25(20.8 \%)$ & $89(29.7 \%)$ & $52(16.1 \%)$ & $181(30.4 \%)$ & $158(15.4 \%)$ \\
\hline 2006-2007 & $94(25.5 \%)$ & $29(24.2 \%)$ & $67(22.3 \%)$ & $75(23.2 \%)$ & $153(25.7 \%)$ & $203(19.8 \%)$ \\
\hline 2008-2010 & $219(59.4 \%)$ & $66(55.0 \%)$ & $144(48.0 \%)$ & $197(60.8 \%)$ & $261(43.9 \%)$ & $667(64.8 \%)$ \\
\hline \multicolumn{7}{|l|}{ Pathological type } \\
\hline Invasive ductal & $347(94.0 \%)$ & $113(94.2 \%)$ & $275(91.7 \%)$ & $304(93.8 \%)$ & $553(92.9 \%)$ & $970(94.4 \%)$ \\
\hline Invasive lobular & $13(3.5 \%)$ & $3(2.5 \%)$ & $2(0.7 \%)$ & $3(0.9 \%)$ & $7(1.2 \%)$ & $8(0.8 \%)$ \\
\hline Other & $9(2.4 \%)$ & $4(3.3 \%)$ & $23(7.7 \%)$ & $17(5.2 \%)$ & $35(5.9 \%)$ & $50(4.8 \%)$ \\
\hline
\end{tabular}

$B C S$ breast-conserving surgery, $E R$ estrogen receptor, $P R$ progesterone receptor, $b o t h *$ chemotherapy + trastuzumab 
and increased by $12.9 \%$, resulting in $57.8 \%$ for $\mathrm{T} 1 \mathrm{~b}$; and increased by $20.0 \%$, resulting in $71.9 \%$ for T1c (Table 1 ). In 2008, the use of trastuzumab was approved in Japan. Thereafter, the administration rates of chemotherapy and/or trastuzumab stabilized at $50-70 \%$ for $\mathrm{T} 1 \mathrm{~b}$ and $\mathrm{T} 1 \mathrm{c}$, and at 20-30\% for T1 a patients, in accordance with the guidelines for the late phase (Fig. 2).

There was no difference in DFS for treated versus untreated patients in either the T1a or T1b groups; however, in the T1c group, treated patients showed significantly better DFS compared with untreated patients (logrank test; $p<0.001$ ) (Fig. 3).

In the Cox proportional hazards model with the background factors adjusted, the treated group had significantly longer survival than the untreated group (T1b: Hazard ratio (HR) 0.20 ; $95 \%$ Confidence interval (CI) $0.06-0.67$ and T1c: HR 0.54; 95\% CI 0.31-0.94) (Table 2). In the multivariate regression model for DFS and OS, the prognosis of treated patients was significantly better compared with untreated patients in the T1c group (DFS: HR 0.47 ; 95\% CI $0.32-0.68$ and OS: HR 0.54, 95\% CI 0.31-0.94). Furthermore, treated patients had significantly better survival compared with untreated patients in the T1b group (BCSS: HR 0.17; 95\% CI $0.03-0.95$ and OS: HR 0.20; 95\% CI 0.06-0.67). Patients with ER - tumors had significantly poorer DFS (HR 2.01, 95\% CI 1.27-3.16), BCSS (HR 3.93; 95\% CI 1.41-11.0), and OS (HR 2.58; 95\% CI 1.27-5.24) compared with patients with ER+tumors in the T1c group. Furthermore, in the T1b group, the hazard of patients aged 35 years or younger was significantly higher compared to the patients aged 50-69 years (HR 3.18 (95\% CI 1.08-9.34)) (Table 2).

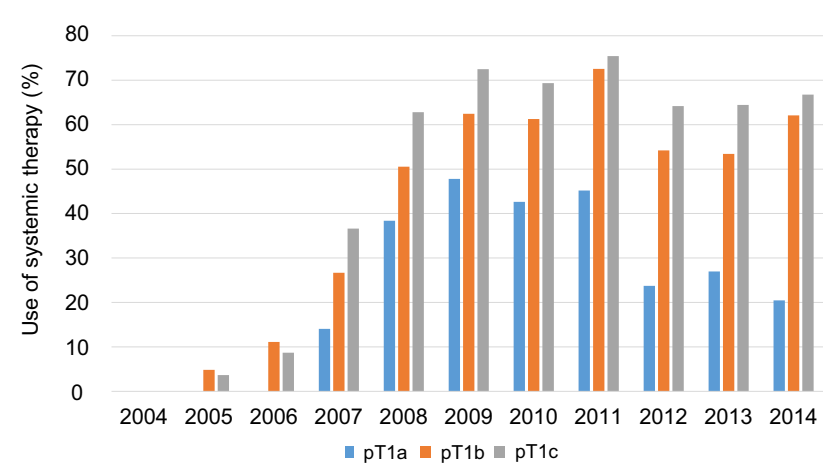

Fig. 2 Use of systemic therapy for HER2 + breast cancer in Japan between 2004 and 2014

\section{Discussion}

In our study using retrospectively collected data from the Japanese BCR held within the NCD, we identified 2736 patients with early-stage pT1N0 HER2+ breast cancer and demonstrated significantly improved survival benefits (DFS: HR 0.45; 95\% CI 0.32-0.68 and OS: HR 0.54; 95\% CI 0.31-0.94) in those who received systemic treatment in the pT1c group (Table 2). In addition, systemic treatment improved outcomes in patients with pT1b disease (BCSS: HR 0.17 ; 95\% CI $0.03-0.95$ and OS: HR 0.20 ; $95 \%$ CI 0.06-0.67). Therefore, our study contributes to our understanding of whether to administer chemotherapy and/or trastuzumab to an increasing population of patients with earlystage HER2+ tumors. Such patients have generally not been included in randomized trials of adjuvant systemic therapy, and presently, there are no randomized trials that can provide level I evidence. Therefore, our observational registry study likely represents the best available and appropriate evidence on the management of benefit and harm in patients with pT1N0 HER2+ breast cancer.

Our data showed that treatment of pT1a tumors was not associated with an improved prognosis; therefore, caution should be used when deciding whether to treat patients with pT1a tumors. Whether all patients with early-stage HER2+ tumors require both adjuvant chemotherapy and 1 year of trastuzumab is still unclear. Guidelines from the National Comprehensive Cancer Network (NCCN) suggest that adjuvant chemotherapy with trastuzumab should be considered in patients with pT1abN0 (or N1mi) HER2+ tumors [24]. van Ramshorst et al., using data from the Netherlands Cancer Registry, reported that treatment benefits in patients given adjuvant chemotherapy and/or trastuzumab were similar in all three early-stage breast tumor groups, with 8-year BCSS estimates of 100 versus (vs.) $95 \%$ in T1a (HR 0.05; $95 \%$ CI $0-8.81 \times 10^{3} ; p=0.62$ ), 99 vs. $94 \%$ in T1b (HR 0.25 ; $95 \%$ CI $0.03-1.88 ; p=0.18$ ), and 96 vs. $90 \%$ in T1c tumors (HR $0.34 ; 95 \%$ CI $0.22-0.52 ; p<0.001$ ), and OS estimates of 100 vs. $85 \%$ in T1a (HR 0.05 ; $95 \%$ CI $0-1.99 \times 10^{2}$; $p=0.47$ ), 99 vs. $89 \%$ in T1b (HR 0.14 ; $95 \%$ CI $0.02-0.99$; $p=0.05$ ), and 94 vs. $80 \%$ in T1c tumors (HR $0.23 ; 95 \% \mathrm{CI}$ $0.16-0.33 ; p<0.001)$ [25]. Tolaney et al. published that in the APT phase II clinical trial, 12 weeks of paclitaxel + trastuzumab followed by 1 year of trastuzumab monotherapy in node-negative HER2+tumors less than $3 \mathrm{~cm}$ (with 18.9\% and $30.5 \%$ of patients having $\mathrm{T} 1 \mathrm{mi} / \mathrm{a}$ and $\mathrm{T} 1 \mathrm{~b}$ tumors, respectively), demonstrated excellent survival outcomes (DFS 93.3\% and OS 95.0\% at 7 years) [26, 27]. Furthermore, they recently described a low incidence of grade 3 to 4 left ventricular systolic dysfunction $(0.5 \%)$ and left ventricular ejection fraction decline $(3.2 \%)$ during treatment with 4-year median follow-up [28]. However, long-term toxic 

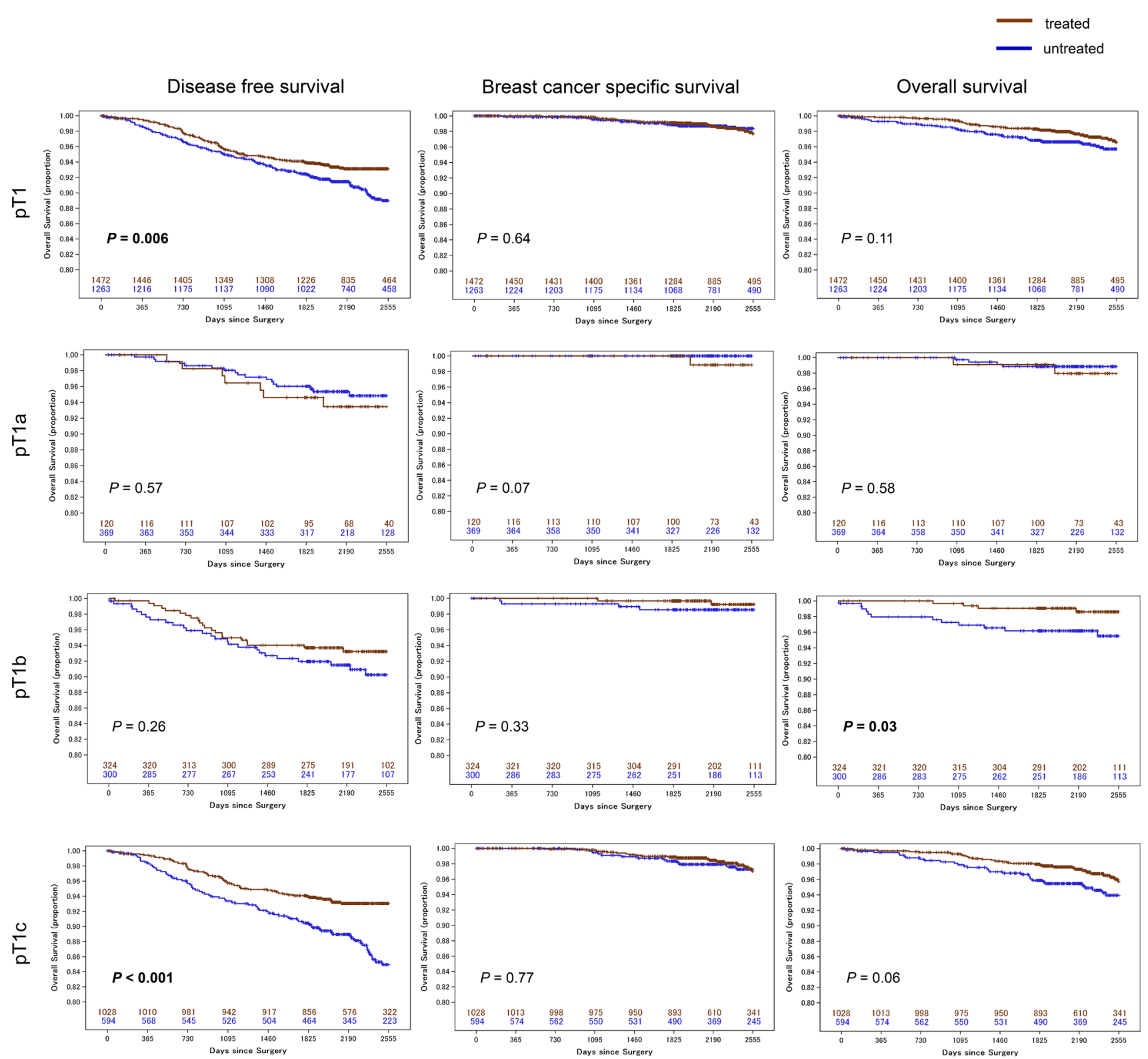

Fig. 3 Survival curves according to systemic therapy status

effects should be taken into consideration in the final decision process. In light of results of our study, our opinion is that treatment of patients with pT1ab HER2+ tumors with trastuzumab-based adjuvant therapy should be discussed.

Our study showed that younger patients ( $<35$ years) even with pT1b tumors had a poor prognosis, as shown in Table 2 (DFS: HR 3.18; 95\% CI 1.08-9.34; $p=0.036$ ). Therefore, we may consider treating younger patients with $\mathrm{pT} 1 \mathrm{~b}$ tumors because there was no difference regarding treatment ratios among the subgroups defined according to age at diagnosis except for the $>70$ years subgroup (Supplementary Fig. 3B). Moreover, elderly patients ( $>70$ years) with pT1c tumors had significantly poorer prognoses (DFS: HR 1.63;
95\% CI $1.06-2.49 ; P=0.026$, and OS: HR $2.49 ; 95 \%$ CI $1.37-4.51 ; p=0.003)$ and, therefore providing treatment to elderly patients is expected to have a significant beneficial survival effect. Sawaki et al. reported in a randomized controlled trial (N-SAS BC07/RESPECT) that trastuzumab monotherapy could be an option as an adjuvant therapy for elderly (70-80 years) HER $2+$ breast cancer patients in light of less toxicity and a better quality of life compared with chemotherapy plus trastuzumab [29]. Of the 266 patients, $49 \%$ were $\mathrm{T} 1 \mathrm{~b}$ and T1c, and DFS at 3 years was $94.8 \%$ in the chemotherapy plus trastuzumab-treated group and $89.2 \%$ in the trastuzumab-treated group (HR 1.42; 95\% CI, 0.68-2.95; $p=0.35$ ). Based on our results, we suggest that the same 
Table 2 Multivariate analysis of 5-year survival

\begin{tabular}{|c|c|c|c|c|c|c|c|c|c|c|c|c|}
\hline \multirow[t]{2}{*}{ Variable } & \multicolumn{4}{|l|}{ DFS } & \multicolumn{4}{|l|}{ BCSS } & \multicolumn{4}{|l|}{ OS } \\
\hline & HR & \multicolumn{2}{|l|}{$95 \% \mathrm{CI}$} & $p$ value & HR & \multicolumn{2}{|l|}{$95 \% \mathrm{CI}$} & $p$ value & HR & \multicolumn{2}{|c|}{$95 \% \mathrm{CI}$} & $p$ value \\
\hline \multicolumn{13}{|l|}{ pT1 } \\
\hline With treatment & 0.677 & 0.508 & 0.901 & 0.0076 & 0.906 & 0.471 & 1.742 & 0.088 & 0.695 & 0.442 & 1.094 & 0.1163 \\
\hline \multicolumn{13}{|l|}{ Age at diagnosis } \\
\hline$<35$ & 2.35 & 1.33 & 4.151 & 0.0033 & 3.834 & 1.099 & 13.369 & 4.4465 & 2.039 & 0.719 & 5.787 & 0.1806 \\
\hline $35-49$ & 0.988 & 0.686 & 1.421 & 0.9463 & 1.625 & 0.737 & 3.583 & 1.4479 & 0.827 & 0.428 & 1.599 & 0.5724 \\
\hline $50-69$ & $(\operatorname{Ref})$ & & & & $(\operatorname{Ref})$ & & & & (Ref) & & & \\
\hline$\geq 70$ & 1.844 & 1.287 & 2.643 & 0.0009 & 2.089 & 0.904 & 4.829 & 2.969 & 2.84 & 1.724 & 4.678 & $<0.0001$ \\
\hline \multicolumn{13}{|l|}{ ER } \\
\hline Negative & 1.561 & 1.083 & 2.248 & 0.0168 & 4.24 & 1.531 & 11.742 & 7.7255 & 2.036 & 1.12 & 3.702 & 0.0197 \\
\hline \multicolumn{13}{|l|}{ PR } \\
\hline Negative & 0.958 & 0.644 & 1.424 & 0.8304 & 0.966 & 0.312 & 2.993 & 0.0036 & 1.12 & 0.562 & 2.23 & 0.7477 \\
\hline \multicolumn{13}{|l|}{ pT1a } \\
\hline With treatment & 1.286 & 0.509 & 3.249 & 0.5945 & 1.575 & 0.272 & 9.109 & 0.9992 & 1.575 & 0.272 & 9.109 & 0.6119 \\
\hline \multicolumn{13}{|l|}{ Age at diagnosis } \\
\hline$<35$ & 1.909 & 0.386 & 9.447 & 0.4279 & 0 & 0 & 0 & 1 & 0 & 0 & 0 & 0.9968 \\
\hline $35-49$ & 1.221 & 0.481 & 3.096 & 0.6743 & 0.475 & 0.05 & 4.517 & 0.9989 & 0.475 & 0.05 & 4.517 & 0.5171 \\
\hline $50-69$ & (Ref) & & & & (Ref) & & & & (Ref) & & & \\
\hline$\geq 70$ & 0.775 & 0.098 & 6.112 & 0.809 & 0 & 0 & 0 & 1 & 0 & 0 & 0 & 0.9959 \\
\hline \multicolumn{13}{|l|}{ ER } \\
\hline Negative & 1.406 & 0.399 & 4.947 & 0.5959 & 0.817 & 0.099 & 6.731 & 0.999 & 0.817 & 0.099 & 6.731 & 0.8511 \\
\hline \multicolumn{13}{|l|}{ PR } \\
\hline Negative & 0.46 & 0.118 & 1.786 & 0.2615 & 0.868 & 0.056 & 13.51 & 0.9996 & 0.868 & 0.056 & 13.51 & 0.9193 \\
\hline \multicolumn{13}{|l|}{ pT1b } \\
\hline With treatment & 0.538 & 0.288 & 1.007 & 0.0526 & 0.169 & 0.03 & 0.947 & 0.0432 & 0.202 & 0.061 & 0.666 & 0.0086 \\
\hline \multicolumn{13}{|l|}{ Age at diagnosis } \\
\hline$<35$ & 3.177 & 1.081 & 9.335 & 0.0355 & 0 & 0 & 0 & 0.9986 & 2.691 & 0.335 & 21.613 & 0.3516 \\
\hline $35-49$ & 1.152 & 0.523 & 2.54 & 0.7252 & 1.723 & 0.177 & 16.812 & 0.6396 & 0.431 & 0.052 & 3.552 & 0.434 \\
\hline $50-69$ & (Ref) & & & & (Ref) & & & & (Ref) & & & \\
\hline$\geq 70$ & 1.476 & 0.658 & 3.31 & 0.3452 & 2.714 & 0.438 & 16.802 & 0.2831 & 2.1 & 0.69 & 6.396 & 0.1914 \\
\hline \multicolumn{13}{|l|}{ ER } \\
\hline Negative & 1.523 & 0.734 & 3.157 & 0.2583 & 0 & 0 & 0 & 0.996 & 2.604 & 0.728 & 9.314 & 0.141 \\
\hline \multicolumn{13}{|l|}{ PR } \\
\hline Negative & 2.249 & 0.936 & 5.403 & 0.0699 & 0 & 0 & 0 & 0.997 & 2.753 & 0.461 & 16.433 & 0.2666 \\
\hline \multicolumn{13}{|l|}{ pT1c } \\
\hline With treatment & 0.468 & 0.323 & 0.68 & $<0.0001$ & 0.572 & 0.265 & 1.237 & 0.1558 & 0.541 & 0.31 & 0.944 & 0.0305 \\
\hline Age at diagnosis & & & & & & & & & & & & \\
\hline$<35$ & 2.174 & 1.031 & 4.584 & 0.0414 & 4.669 & 1.295 & 16.84 & 0.0186 & 2.229 & 0.663 & 7.491 & 0.1952 \\
\hline $35-49$ & 0.911 & 0.573 & 1.446 & 0.6913 & 1.912 & 0.811 & 4.506 & 0.1384 & 1.03 & 0.49 & 2.165 & 0.9379 \\
\hline $50-69$ & (Ref) & & & & (Ref) & & & & (Ref) & & & \\
\hline$\geq 70$ & 1.626 & 1.061 & 2.489 & 0.0255 & 1.394 & 0.523 & 3.717 & 0.5067 & 2.487 & 1.371 & 4.51 & 0.0027 \\
\hline ER & & & & & & & & & & & & \\
\hline Negative & 2.005 & 1.273 & 3.158 & 0.0027 & 3.933 & 1.41 & 10.969 & 0.0089 & 2.578 & 1.269 & 5.237 & 0.0088 \\
\hline PR & & & & & & & & & & & & \\
\hline Negative & 0.912 & 0.564 & 1.475 & 0.7065 & 1.194 & 0.384 & 3.716 & 0.7591 & 1.078 & 0.49 & 2.372 & 0.8511 \\
\hline
\end{tabular}

$B C S S$ breast cancer-specific survival, $C I$ confidence interval, $D F S$ disease-free survival, $E R$ estrogen receptor, $H R$ hazard ratio, $O S$ overall survival, $P R$ progesterone receptor: treatment, chemotherapy and/or trastuzumab, ref reference 
treatment options should be discussed for all patients with pT1N0 HER2+ tumors, regardless of tumor size or age.

Our data showed that patients with pT1c tumors that were ER- had a poor prognosis (DFS: HR 2.01; 95\% CI 1.27-3.16; $p=0.0027$, BCSS: HR 3.93; 95\% CI 1.41-11.0; $p=0.0089$, and OS: HR $2.58 ; 95 \%$ CI $1.27-5.24$, $p=0.0088$ ). However, regarding $\mathrm{pT} 1 \mathrm{ab}$ tumors, being ER- was not a predictive marker. In Japan, physicians tend to avoid adjuvant chemotherapy for patients with ER+/ HER2+tumors (Supplementary Fig. 3A). van Ramshorst et al. demonstrated that improved OS and BCSS outcomes were seen with systemic treatment in both hormone receptor-positive and -negative subgroups with a relative risk reduction in the same range, but that the absolute benefit was smaller in the hormone receptor-positive subgroup [25]. The difference can be explained by the use of adjuvant endocrine therapy for both hormone receptor-positive subgroups, regardless of chemotherapy and/or trastuzumab treatment. According to a meta-analysis of the randomized trial data by O'Sullivan et al., addition of trastuzumab to the chemotherapy improved DFS and OS in 4220 patients with early-stage HER2+ tumors, half of whom had none or one positive lymph node, and the proportional benefit was similar in both hormone receptor-positive and -negative diseases [30]. In the APT trial, the 3-year rate of recurrence-free survival (RFS) was 99.2\% (95\% CI 98.4-100), and there was no difference in subgroups defined according to tumor size $(\leq 1 \mathrm{~cm}$ vs. $>1 \mathrm{~cm}$ ) or hormone receptor status (positive vs. negative) [27]. Therefore, our viewpoint is that treatment with chemotherapy and/or trastuzumab for patients with pT1c tumors, particularly those that are ER-, is warranted.

The use of endocrine therapy combined with HER2-targeted therapy implies a type of dual blockade for patients with ER+/HER2+ tumors. Dackus et al. have recently published that aromatase inhibitors (AIs) (with or without ovarian ablation) were associated with better RFS and OS outcomes in ER+/HER2+ perimenopausal (aged 45-55 years) breast cancer patients, using data from the NCR [31]. According to multivariate Cox regression analysis, premenopausal women derived a significant survival benefit from AIs compared with tamoxifen (RFS: HR 0.47; 95\% CI $0.25-0.91, p=0.03$ and OS: HR 0.37 ; 95\% CI $0.18-0.79$, $p=0.01$ ). It is essential to use endocrine therapy combined with HER2-targeted therapy concurrently or sequentially. Moreover, an antibody-drug conjugate of trastuzumab and the toxic agent, trastuzumab-emtansine (T-DM1), is expected to replace trastuzumab-based treatment in various clinical situations. The phase II ATEMPT trial is ongoing, and patients with stage I HER2+ tumors are assigned to receive paclitaxel and trastuzumab followed by 1 year of trastuzumab or T-DM1 [32]. von Minckwitz et al. have recently published that the risk of recurrence of invasive breast cancer or death was 50\% lower with adjuvant T-DM1 than with adjuvant continuation of trastuzumab alone for patients with HER2+early breast cancer who had residual invasive disease after completion of neoadjuvant therapy [33]. Adverse events of grade 3 or higher were more common in the T-DM1 than in the trastuzumab group ( $25.7 \mathrm{vs.}$ $15.4 \%$ ). In light of the above, careful patient selection is most important when making decisions on a treatment regimen to avoid unnecessary toxicity.

There are several important limitations to this study. First, it consisted of retrospectively collected cases, including the possibility of selection and precluding the determination of causal relationships. However, Japanese BCR data cover more than $50 \%$ of breast cancer patients diagnosed in Japan. Therefore, we do not expect that selection would substantially affect our findings. Second, data on histological grade and Ki-67 labeling index were not available. There was the possibility of unmeasured confounding regarding this point. Finally, our data were not centrally reassessed on ER, PR, or HER2 status. However, the strength of our study is that it draws from more than 50,000 patients treated by qualified doctors and institutions in a 'real-world' setting and that it has internal and external validity. Our goal is to be able to identify patients with early-stage HER2+ tumors who require systemic treatment including HER2-targeted agents.

\section{Conclusions}

In conclusion, our findings demonstrate that systemic treatment and/or trastuzumab may not be necessary in the clinical management of pT1a HER2+tumors. However, these treatments may be beneficial for pT1b tumors compared with no treatment. These results are compatible with various guidelines such as of those of the NCCN. Additional research using clinical registry data may be essential for verifying guideline treatments for this subgroup of breast cancer patients.

Acknowledgements We would like to thank Sheryl Valencia, MD, assistant professor of Kyushu University Hospital, and Mark Abramovitz, $\mathrm{PhD}$, from Edanz Group (www.edanzediting.com/ac) for English language editing.

Author contributions Study concept and design: MK (Makoto Kubo), MK (Masaaki Kawai), and HK. Assembly of data: HK. Manuscript writing: MK (Makoto Kubo), MK (Masaaki Kawai), and HK. Critical revision of the manuscript for important intellectual content: The Registration Committee of the JBCS (KT, MY, EO, MN, SA, YK, TK, KA, NN, MM, KI, NH, YY, SI, and HJ). Final approval of manuscript: SI and HJ.

Funding This work was funded by the Registration Committee of the Japanese Breast Cancer Society (Grant Number: 2015-1). 


\section{Compliance with ethical standards}

Conflict of interest Each author of $\mathrm{NH}$ and $\mathrm{YY}$ has received honorariums as a speaker or consultant/advisory role from Company Chugai Pharmaceutical Co. (Tokyo, Japan). The other authors declare that they have no conflicts of interest.

Ethical approval This article does not contain any studies with animals performed by any of the authors. All procedures performed in studies involving human participants were in accordance with the ethical standards of the institutional and/or national research committee and with the 1964 Helsinki declaration and its later amendments or comparable ethical standards.

Informed consent Informed consent was obtained from all individual participants included in the study. The study was approved by the Research Ethics Committee of NCD/JBCS and Kyushu University Hospital (No. 30-450).

Open Access This article is distributed under the terms of the Creative Commons Attribution 4.0 International License (http://creativeco mmons.org/licenses/by/4.0/), which permits unrestricted use, distribution, and reproduction in any medium, provided you give appropriate credit to the original author(s) and the source, provide a link to the Creative Commons license, and indicate if changes were made.

\section{References}

1. Japanese Breast Cancer Society: http://jbcs.gr.jp/member/en/. Accessed 21 Feb 2019

2. Kennedy T, Stewart AK, Bilimoria KY et al (2007) Treatment trends and factors associated with survival in T1aN0 and T1bN0 breast cancer patients. Ann Surg Oncol 14:2918-2927

3. Berry DA, Cronin KA, Plevritis SK et al (2005) Effect of screening and adjuvant therapy on mortality from breast cancer. N Engl J Med 353:1784-1792

4. Van Maaren MC, Strobbe LJA, Smidt ML et al (2018) Ten-year conditional recurrence risks and overall and relative survival for breast cancer patients in the Netherlands: taking account of eventfree years. Eur J Cancer 102:82-94

5. Schroeder MC, Lynch CF, Abu-Hejleh T et al (2015) Chemotherapy use and surgical treatment by receptor subtype in nodenegative T1a and T1b female breast cancers, Iowa SEER Registry, 2010 to 2012. Clin Breast Cancer 15:e27-e34

6. Gonzalez-Angulo AM, Litton JK, Broglio KR et al (2009) High risk of recurrence for patients with breast cancer who have human epidermal growth factor receptor 2-positive, node-negative tumors $1 \mathrm{~cm}$ or smaller. J Clin Oncol 27:5700-5706

7. Parker JS, Mullins M, Cheang MC et al (2009) Supervised risk predictor of breast cancer based on intrinsic subtypes. J Clin Oncol 27:1160-1167

8. Hudis CA (2007) Trastuzumab-mechanism of action and use in clinical practice. N Engl J Med 357:39-51

9. Curigliano G, Viale G, Bagnardi V et al (2009) Clinical relevance of HER2 overexpression/amplification in patients with small tumor size and node-negative breast cancer. J Clin Oncol 27:5693-5699

10. Gianni L, Dafni U, Gelber RD et al (2011) Treatment with trastuzumab for 1 year after adjuvant chemotherapy in patients with HER2+ early breast cancer: a 4-year follow-up of a randomised controlled trial. Lancet Oncol 12:236-244

11. Joensuu H, Bono P, Kataja V et al (2009) Fluorouracil, epirubicin, and cyclophosphamide with either docetaxel or vinorelbine, with or without trastuzumab, as adjuvant treatments of breast cancer: final results of the FinHer Trial. J Clin Oncol 27:5685-5692

12. Perez EA, Romond EH, Suman VJ et al (2011) Four-year follow-up of trastuzumab plus adjuvant chemotherapy for operable human epidermal growth factor receptor 2-positive breast cancer: joint analysis of data from NCCTG N9831 and NSABP B-31. J Clin Oncol 29:3366-3373

13. Slamon D, Eiermann W, Robert N et al (2011) Adjuvant trastuzumab in HER2+ breast cancer. N Engl J Med 365:1273-1283

14. Spielmann M, Roché H, Delozier T et al (2009) Trastuzumab for patients with axillary-node-positive breast cancer: results of the FNCLCC-PACS 04 trial. J Clin Oncol 27:6129-6134

15. Perez EA, Romond EH, Suman VJ et al (2014) Trastuzumab plus adjuvant chemotherapy for human epidermal growth factor receptor 2-positive breast cancer: planned joint analysis of overall survival from NSABP B-31 and NCCTG N9831. J Clin Oncol 32:3744-3752

16. Cameron D, Piccart-Gebhart MJ, Gelber RD et al (2017) 11 years' follow-up of trastuzumab after adjuvant chemotherapy in HER2+ early breast cancer: final analysis of the HERceptin Adjuvant (HERA) trial. Lancet 389:1195-1205

17. Sobin LH, Gospodarowicz MK, Wittekind C (2010) TNM classification of malignant tumours, 7th edn. Wiley, New York, pp 131-141

18. The Japanese Breast Cancer Society (2012) General rules for clinical and pathological recording of breast cancer, $17 \mathrm{th}$ edn. Kanehara Shuppan, Tokyo

19. Lakhani S, Ellis I, Schnitt S et al (2012) WHO classification of tumours of the breast, 4 th edn. IARC Press, Lyon

20. Wolff AC, Hammond ME, Schwartz JN et al (2007) American Society of Clinical Oncology/College of American Pathologists guideline recommendations for human epidermal growth factor receptor 2 testing in breast cancer. Arch Pathol Lab Med 131:18-43

21. Prat A, Cheang MC, Martín M et al (2013) Prognostic significance of progesterone receptor-positive tumor cells within immunohistochemically defined luminal A breast cancer. J Clin Oncol 31:203-209

22. Goldhirsch A, Winer EP, Coates AS et al (2013) Personalizing the treatment of women with early breast cancer: highlights of the St Gallen International Expert Consensus on the Primary Therapy of Early Breast Cancer 2013. Ann Oncol 24:2206-2223

23. Coates AS, Winer EP, Goldhirsch A et al (2015) Tailoring therapies-improving the management of early breast cancer: St Gallen International Expert Consensus on the Primary Therapy of Early Breast Cancer 2015. Ann Oncol 26:1533-1546

24. National Comprehensive Cancer Network. http://www.nccn.org. Accessed 21 Feb 2019

25. Van Ramshorst MS, Dackus GM et al (2016) The effect of trastuzumab-based chemotherapy in small node-negative HER2+ breast cancer. Breast Cancer Res Treat 158:361-371

26. Tolaney SM, Barry WT, Dang CT et al (2015) Adjuvant paclitaxel and trastuzumab for node-negative, HER2-positive breast cancer. N Engl J Med 372:134-141

27. Tolaney SM, Barry WT, Guo H et al (2017) Seven-year (yr) follow-up of adjuvant paclitaxel (T) and trastuzumab (H) (APT trial) for node-negative, HER2-positive breast cancer (BC). J Clin Oncol. https://doi.org/10.1200/JCO.2017.35.15_suppl.511

28. Dang C, Guo H, Najita J et al (2016) Cardiac outcomes of patients receiving adjuvant weekly paclitaxel and trastuzumab for nodenegative, ERBB2-positive breast cancer. JAMA Oncol 2:29-36

29. Sawaki M, Saito T, Baba S et al (2018) Evaluation of trastuzumab without chemotherapy as a postoperative adjuvant therapy in HER2-positive elderly breast cancer patients: randomized controlled trial (RESPECT). J Clin Oncol. https://doi.org/10.1200/ JCO.2018.36.15_suppl.510 
30. O'Sullivan CC, Bradbury I, Campbell C et al (2015) Efficacy of adjuvant trastuzumab for patients with human epidermal growth factor receptor 2-positive early breast cancer and tumors $\leq 2 \mathrm{~cm}$ : a meta-analysis of the randomized trastuzumab trials. J Clin Oncol 33:2600-2608

31. Dackus GMHE, Jóźwiak K, Sonke GS et al (2018) Optimal adjuvant endocrine treatment of ER+/HER2+ breast cancer patients by age at diagnosis: a population-based cohort study. Eur J Cancer 90:92-101

32. ClinicalTrials.gov: T-DM1 vs paclitaxel/trastuzumab for breast (ATEMPT Trial). https://clinicaltrials.gov/ct2/show/NCT0185374 8. Accessed 21 Feb 2019
33. Von Minckwitz G, Huang CS, Mano MS et al (2019) Trastuzumab emtansine for residual invasive her2-positive breast cancer. N Engl J Med 380:617-628

Publisher's Note Springer Nature remains neutral with regard to jurisdictional claims in published maps and institutional affiliations.

\section{Affiliations}

\section{Makoto Kubo $^{1}$ D $\cdot$ Masaaki Kawai ${ }^{2} \cdot$ Hiraku Kumamaru $^{3} \cdot$ Hiroaki Miyata $^{3} \cdot K_{\text {Kenji Tamura }}{ }^{4}$ Masayuki Yoshida $^{5}$. Etsuyo Ogo ${ }^{6} \cdot$ Masayuki Nagahashi $^{7}$. Sota Asaga ${ }^{8} \cdot$ Yasuyuki Kojima $^{9} \cdot$ Takayuki Kadoya $^{10} \cdot$ Kenjiro Aogi $^{11}$. Naoki Niikura ${ }^{12} \cdot$ Minoru Miyashita $^{13} \cdot$ Kotaro lijima $^{14} \cdot$ Naoki Hayashi $^{15} \cdot$ Yutaka Yamamoto $^{16} \cdot$ Shigeru Imoto $^{17}$. Hiromitsu Jinno ${ }^{18}$}

1 Department of Surgery and Oncology, Graduate School of Medical Sciences, Kyushu University, 3-1-1 Maidashi Higashi-ku, Fukuoka 812-8582, Japan

2 Department of Breast Oncology, Miyagi Cancer Center Hospital, 47-1 Nodayama, Medeshima-Shiode, Natori, Miyagi 981-1293, Japan

3 Department of Healthcare Quality Assessment, University of Tokyo, 7-3-1 Hongo, Bunkyo-ku, Tokyo 113-8655, Japan

4 Department of Breast and Medical Oncology, National Cancer Center Hospital, 5-1-1 Tsukiji, Chuo-ku, Tokyo 104-0045, Japan

5 Department of Diagnostic Pathology, National Cancer Center Hospital, 5-1-1 Tsukiji, Chuo-ku, Tokyo 104-0045, Japan

6 Department of Radiology, Kurume University School of Medicine, 67 Asahi-Machi, Kurume, Fukuoka 830-0011, Japan

7 Division of Digestive and General Surgery, Niigata University Graduate School of Medical and Dental Sciences, 1-757 Asahimachi-dori, Chuo-ku, Niigata 951-8510, Japan

8 Department of Breast Surgery, Kyorin University School of Medicine, 6-20-2 Shinkawa, Mitaka, Tokyo 181-8611, Japan

9 Division of Breast and Endocrine Surgery, Department of Surgery, St. Marianna University School of Medicine, 2-16-1 Sugao, Miyamae-ku, Kawasaki 216-8511, Japan
10 Department of Surgical Oncology, Research Institute for Radiation Biology and Medicine, Hiroshima University, 1-2-3 Kasumi, Minami-ku, Hiroshima 734-0037, Japan

11 Department of Breast Oncology, National Hospital Organization Shikoku Cancer Center, Kou 160, Minamiumemotomachi, Matsuyama, Ehime 791-0280, Japan

12 Department of Breast and Endocrine Surgery, Tokai University School of Medicine, 143 Shimokasuya, Isehara, Kanagawa 259-1193, Japan

13 Department of Breast and Endocrine Surgical Oncology, Tohoku University School of Medicine, Seiryo-machi, Aoba-ku, Sendai 980-8574, Japan

14 Department of Breast Oncology, Juntendo University, 3-1-3 Hongo, Bunkyo-ku, Tokyo 113-8431, Japan

15 Department of Breast Surgical Oncology, St. Luke's International Hospital, 9-1 Akashicho, Chuo-ku, Tokyo 104-8560, Japan

16 Department of Molecular-Targeting Therapy for Breast Cancer, Kumamoto University, 1-1-1 Honjo, Chuo-ku, Kumamoto 860-8556, Japan

17 Department of Breast Surgery, Kyorin University Hospital, 6-20-2 Shinkawa, Mitaka, Tokyo 181-8611, Japan

18 Department of Surgery, Teikyo University School of Medicine, 2-11-1 Kaga, Itabashi-ku, Tokyo 173-8606, Japan 